\title{
The Relation Between Disinhibition and Emotion Regulation in Boys With Attention Deficit Hyperactivity Disorder
}

\author{
Christy Mangione Walcott and Steven Landau \\ Department of Psychology, Illinois State University
}

\begin{abstract}
This study examined group differences of 49 boys ages 6 to 11 years with and without attention deficit hyperactivity disorder $(A D H D)$ in emotion regulation during frustrating peer competition. Half of all boys in each group were explicitly instructed to hide their feelings if they became upset during the competition. Behavioral inhibition, both before and after the competitive task, was examined using the Stop Signal Task (SST), and emotion regulation was assessed via structured observation data. Effect sizes indicated that impulsive ADHD boys displayed greater disinhibition and were less effective at emotion regulation than comparison boys. In addition, boys with ADHD were unsuccessful in masking their emotions even when instructed to do so. In contrast, comparison boys were more successful at emotion regulation when given instruction to self-regulate, and these regulatory attempts predicted later inhibitory control. Findings are discussed in the context of current ADHD-related theories of inhibitory deficit, and suggestions for future research are provided.
\end{abstract}

Disturbed peer relationships are common among children with attention deficit hyperactivity disorder (ADHD). These children tend to be easily frustrated, emotionally explosive, and less attentive to social cues (Henker \& Whalen, 1999; Landau, Milich, \& Diener, 1998). Peers often consider children with ADHD to be aversive playmates because of their tendency toward behavioral excess and impulsive responding. Despite controversy regarding whether children with ADHD possess necessary social skills required to be successful playmates (Guevremont \& Dumas, 1994), there is little doubt they experience difficulty employing the social skills they do possess. Such performance deficits may result directly from poor inhibitory control. This, in turn, hinders their ability to delay responding long enough to consider the intricacies of a social situation and to access their repertoire of social skills (Barkley, 1997).

This focus on impulsivity and executive control in relation to ADHD has driven current theoretical models (Barkley, 1997; Logan, Schachar, \& Tannock, 1997; Pennington \& Ozonoff, 1996; Quay, 1997). For example, Barkley's theory implicates ADHD-related deficits in three processes of behavior inhibition: (a) stopping initial prepotent responses to an event, (b) stopping an ongoing response to permit a delay for behavioral decision making, and (c) protecting this

Christy Mangione Walcott is now at East Carolina University, Greenville, North Carolina.

Requests for reprints should be sent to Christy M. Walcott, Department of Psychology, 314 Rawl Building, East Carolina University, Greenville, NC 27858. E-mail: walcottc@mail.ecu.edu period of delay from disruption by competing events and responses (i.e., interference control). According to Barkley, because children with ADHD exhibit behavioral disinhibition, they consequently display deficits in apparent cognitive abilities governed by this delay. Thus, when frustrated, children with ADHD may not effectively use self-regulation strategies because their disinhibition precludes the necessary delay for doing so.

Compelling evidence supports behavioral disinhibition as a defining characteristic of ADHD (Barkley, 1997; Nigg, 1999; Oosterlaan, Logan, \& Sergeant, 1998; Pennington \& Ozonoff, 1996; Schachar, Mota, Logan, Tannock, \& Klim, 2000). However, questions remain regarding the exact relation between ADHD and inhibition and the exact processes being tapped by various measures of inhibition (see Nigg, 2001, for a detailed review). For example, Nigg, Blaskey, HuangPollock, and Rappley (2002) found ADHD subtype differences in behavioral inhibition when examining children with ADHD-Predominantly Inattentive type (ADHD-I) and those with ADHD-Combined type (ADHD-C). All children with combined impulsivity and inattention problems (ADHD-C) displayed deficits compared to controls, but for the ADHD-I group, only girls displayed deficits in behavioral inhibition. Thus, subtypes of ADHD, at least among boys, may be discernible on the basis of behavioral disinhibition. In addition, these gender differences reported by Nigg and colleagues provided further evidence that boys and girls with ADHD differ regarding the topography of their executive function difficulties (see also Gaub \& Carlson, 1997). 
Further questions regarding the relation between ADHD and inhibition arise from the broad use of the term inhibition without sufficient consideration of its multifaceted nature (Nigg, 2000, 2001). Nigg (2001) proposed that two distinct types of inhibition be explored in relation to ADHD: motivational and executive. Motivational inhibition is an automatic cessation of an ongoing response that is caused by fear or anxiety resulting from a novel event. In contrast, executive inhibition refers to a process of deliberate suppression of a response for goal-directed purposes. Under this rubric, two different theories of ADHD may both endorse deficits in inhibition (e.g., Barkley, 1997; Quay, 1997) and yet implicate different types of inhibitory dysfunction. Of interest for this study is the connection between executive inhibition, as measured by an established behavioral inhibition task (Stop Signal Task [SST]; Logan, Cowan, \& Davis, 1984), and emotion regulation among children with ADHD.

Emotion regulation is the ongoing process of responding to one's environment with emotions that are both socially acceptable and context-appropriate for a given situation (Cole, Michel, \& Teti, 1994). Therefore, displays of emotion are judged by traditional social norms that guide affective display, as well as by the particular context surrounding the display. It follows, then, that emotion dysregulation may result from either lack of knowledge regarding affective display rules or difficulty modulating emotional reaction in response to social rules or context demands (Cole, Michel, et al., 1994; Saarni, 1999). Barkley's (1997) behavioral disinhibition theory implicates the latter, suggesting that children with ADHD do not effectively delay responding so as to consider social context and display rules separate from the emotional charge of a situation. Because they do not consider vital social cues and rules, they appear more socially dysregulated. According to this view, successful emotion regulation depends on successful behavioral inhibition. The purpose of this research was to examine whether executive behavior inhibition contributes to our understanding of emotion dysregulation in boys with ADHD.

Although the direct relation between executive behavior inhibition and emotion regulation in children with ADHD has not been studied, researchers have found that emotion dysregulation is linked to disruptive behavior in general (e.g., Cole, Zahn-Waxler, \& Smith, 1994; Eisenberg, et al., 1996; Shields \& Cicchetti, 2001; Shields, Cicchetti, \& Ryan, 1994). In many studies of emotion regulation, the construct is considered from a temperamental perspective and measured by global ratings of one's general emotional disposition. For example, Sanson, Smart, Prior, and Oberklaid (1993) found that hyperactive children were rated temperamentally difficult (e.g., socially inflexible, poor attention and concentration, emotionally intense). Similarly, Shields and Cicchetti (2001) mea- sured emotion dysregulation via global ratings of personality characteristics such as reactivity, empathy, arousal, and mood lability and found correlations with disruptive behavior.

Direct behavioral measures have been used to study emotion regulation as well. For example, Cole, ZahnWaxler, et al. (1994) found that preschool children who were ineffective at regulating emotion during a disappointment task were more likely to be rated at-risk for future behavior problems. Hinshaw and Melnick (1995) directly observed emotional reactivity and regulation in boys with ADHD during an unsolvable puzzle task designed to elicit frustration. Boys with ADHD were grouped by high- or low-aggression status, and their emotional display and regulation strategies were coded during the puzzle task. Relative to low-aggressive ADHD and normal comparison boys, boys with ADHD in the high-aggressive group were rated as significantly more emotionally reactive and less effective at emotion regulation. In these investigations, emotional reactivity and dysregulation were not significantly related among low-aggressive boys with ADHD, suggesting it may be aggression and not symptoms of ADHD per se that account for this emotional responding. However, in both studies, the high- and low-aggressive groupings were derived according to former diagnostic criteria (Diagnostic and Statistical Manual of Mental Disorder [3rd ed., rev. $\{D S M-I I I-R\}]$, American Psychiatric Association, 1987), which did not differentiate hyperactive-impulsive and inattentive symptoms (i.e., any 8 of 14 symptoms from the criteria set). As such, the low-aggressive ADHD group in these studies may not have presented the same impulsivity problems as the high-aggressive group and possibly better fit the profile of the Predominantly Inattentive subtype described in the current taxonomy (i.e., Diagnostic and Statistical Manual of Mental Disorders [4th ed. $\{D S M-I V\}]$, American Psychiatric Association, 1994).

In support of this possibility, Maedgen and Carlson (2000) used a procedure comparing children's emotional reactions to both a disappointing and a nondisappointing event and found that children with ADHD-C were rated more intense and less effective at emotion regulation than controls based on global ratings of overall reaction to disappointment. Children with ADHD-I did not differ significantly from either ADHD-C or control children on these global emotion-regulation ratings. When event-coded data were analyzed, there was a nonsignificant trend for children with ADHD-C to display more frequent negative expression of emotion (e.g., a frown or grimace) during the disappointment condition compared to both ADHD-I and non-ADHD children.

The purpose of this study was to extend findings regarding the connection between emotion dysregulation and symptoms of ADHD. The SST (Logan et al., 1984) 
measured executive behavior inhibition before and after a laboratory analog of intense peer competition, which was designed to evoke frustration and elicit emotional reaction. Displays of emotion regulation were coded during the competitive task, and the relation between pretest disinhibition and subsequent emotion regulation was explored. In addition, the effect of boys' attempts at emotion regulation on posttest measures of inhibition was examined. Specifically, one half of all participants were told to hide their feelings if they became upset. To operationalize emotion regulation for this study, two regulation components that had been measured with global ratings in the Hinshaw and Melnick (1995) and Maedgen and Carlson (2000) investigations, emotional reactivity and regulation of affect, were included. Although various subcomponents of emotion regulation are mentioned in the emotion literature (e.g., angry reactivity, emotional intensity, empathy, mood lability, contextual appropriateness), reliable and valid measures of competency in these individual areas are not available (Shields \& Cicchetti, 2001). As such, emotion regulation, for this study, was defined by a composite of global ratings and eventcoded data measuring display of emotional intensity, attempts to modulate emotional display in the presence of a peer, and attempts to maintain task-oriented behavior during a frustrating task.

It was hypothesized that, compared to non-ADHD peers, those with ADHD-C would not be able to effectively regulate their emotions during the frustrating task, as Maedgen and Carlson (2000) reported. This was expected to be true even when boys were given explicit instruction to hide their emotional display in the presence of a peer. Conversely, we predicted that nonsymptomatic boys would better regulate their emotional displays in response to contextual demands and that better regulation would predict more successful inhibitory control.

\section{Method}

\section{Participants}

Forty-nine boys between the ages of 6 and 11 participated in the study; 26 boys were identified as ADHD and 23 were non-ADHD comparison boys. There were 46 Caucasian and 3 African American boys in the sample. Data from 3 boys in the original sample of 52 were discarded because of inability to meet ADHD selection criteria or unreliable performance on the SST. All boys were recruited from a Midwestern school district with total enrollment of approximately 5,800 children across grades pre-K through 12 . Boys alone were selected because ADHD is a male-dominated disorder (American Psychiatric Association, 1994), and there is evidence that boys and girls with ADHD differ regard- ing the topography of their executive function difficulties (Gaub \& Carlson, 1997; Nigg et al., 2002). None of the participants' parents reported any evidence of a pervasive developmental disorder, mental retardation, Tourette's syndrome, seizure disorder, or any other known neurological disorder. All families received \$10 compensation for time and travel inconveniences, and all boys received prizes for their involvement.

\section{Group Assignment}

To recruit potential ADHD participants with significant hyperactive-impulsive symptoms while protecting their anonymity prior to consent, school principals or nurses sent informed-consent letters to the parents of all boys in Grades 1 through 5 who were receiving medication (e.g., methylphenidate or Ritalin) for the treatment of ADHD. Eighty-five informed-consent letters were given to the elementary schools for distribution. The investigators remained unaware of ADHD boys' identities until interested parents actively consented to their child's participation by returning a permission form to the school or contacting the investigators directly. To confirm that each boy's ADHD symptom pattern was consistent with $D S M-I V$ diagnostic criteria for Hyperactive-Impulsive or Combined type, and to confirm these symptoms occurred at a clinically elevated level compared to peers, consenting parents completed the ADHD Rating Scale-IV-Home Version (DuPaul, Power, Anastopoulos, \& Reid, 1998) via interview during the laboratory session. Parents were asked to complete ratings based on their observations of boys' typical behavior over the past 6 months when "he was not on medication." This scale has demonstrated predictive validity and clinical utility in the assessment of ADHD and has been found reliable in its discrimination among ADHD subtypes (Power, Costigan, Leff, Eiraldi, \& Landau, 2001). Boys were assigned to the ADHD group only if they reached or exceeded the 85th percentile cutoff on the Hyperactive-Impulsive or Total scales of the ADHD Rating Scale-IV (DuPaul et al., 1998). Data from those who exceed the 85 th percentile on the Inattention scale only $(n=1)$ were discarded.

Non-ADHD comparison boys, free of special-education diagnoses and medication status, were recruited among same-school grademates in an attempt to match the samples in terms of age and recruitment source. Using the same procedures as with the ADHD group, 150 informed-consent letters describing the study were distributed to parents of same-school grade-mates, and parents were asked to reply if interested in participating. Parents of non-ADHD participants also completed the ADHD Rating Scale-IV via interview, and boys were excluded if scores reached or exceeded the 85 th percentile on any subscale. 
Parent ratings on the ADHD Rating Scale-IV supported experimental groupings: Boys in the ADHD group were rated significantly more hyperactive-impulsive and inattentive than comparison boys (see Table 1). For the ADHD group, the average percentile rank (based on normative data) on the Hyperactive-Impulsive scale was the 93rd percentile (range $=$ 85th to 99th percentile); the average percentile rank on the Inattentive scale was the 90th percentile (range = 85th to 99th percentile). Thus, according to their parents, all boys in the ADHD group displayed symptoms consistent with ADHD-C.

Because psychostimulant medication is known to reduce impulsive responding on the behavioral inhibition measure used in this study (Pliszka, Borcherding, Spratley, Leon, \& Irick, 1997), parents of ADHD participants were instructed to withhold medication for at least $24 \mathrm{hr}$ prior to the laboratory session, based on average duration of action for the most common stimulant medications used to treat ADHD (Pelham, 1993). Forty-six of the 49 participant sessions were scheduled during the weekend, and average elapsed time since last dose ranged from 25 to $28 \mathrm{hr}$. For the single ADHD participant scheduled during the week, the parent brought him in the evening without administering any medication the day of the session. The majority of comparison boys were scheduled on weekends to match ADHD participants, with a few choosing weekday evenings $(n=2)$.

As in the Maedgen and Carlson (2000) study, boys were not excluded due to the presence of comorbid aggression. The decision to include ADHD children who may be comorbid for aggression was logistical, due to small ADHD sample sizes and substantial comorbidity rates of oppositional defiant disorder and conduct disorder in the overall ADHD population (e.g., Biederman, Newcorn, \& Sprich, 1991). In addition, Pliszka and colleagues (1997) reported that stop signal reaction time (SSRT), the measure of behavioral inhibition used in this study, is not significantly correlated with aggression.

Age was not a significant predictor of any dependent variables. In addition, mean age of each group (ADHD: $M=112$ months, $S D=15$; control: $M=108$ months, $S D=12$ ) did not differ significantly, $t(47)=$ $-1.24, p=.22, \eta^{2}=.03$. Therefore, age was not included as a covariate in subsequent analyses.

\section{Measures}

\section{SST}

On the SST (Logan et al., 1984), children with ADHD have consistently displayed significantly less inhibitory control than non-ADHD children (see Oosterlaan et al., 1998, for a meta-analytic review). During the SST, participants pressed a button according to the specified stimulus appearing on the screen. The primary task was to correctly press assigned buttons on the appearance of an $X$ or an $O$. However, the secondary task required that if the $X$ or $O$ occurred after a predetermined signal (i.e., a tone), then participants should not press the assigned button for that trial. In other words, they were expected to suppress or stop their response when the tone sounded. As such, a race was created between reaction time to the primary task (i.e., pressing in response to the $X$ or $O$ ) and the inhibitory process (i.e., withholding primary response when the tone sounded). If one's ability to withhold a response is faster than the reaction time to the primary task, inhibition is successful. However, if the inhibitory process is too slow, participants are unable to stop their ongoing response and react to the primary task erroneously (i.e., error of commission). Participants may also err by withholding a response despite no stop signal tone (i.e., error of omission), and this would be reflected by poor accuracy on the primary task. As suggested by G. D. Logan (personal communication, April 6,2000 ), if a participant's accuracy on the primary discrimination task was less than $80 \%$ accurate $(n=2$ in this study), data were deemed unreliable.

Participant's SST data depicted average performance across four blocks of trials (there were 64 trials per block, and data were averaged across each block). Output data provided by the computer program for each participant included percentage correct on the primary discrimination task, mean reaction time, and

Table 1. ADHD Rating Scale-IV Mean Raw Scores and Pretest Stop Signal Reaction Time by Group

\begin{tabular}{|c|c|c|c|c|c|c|c|}
\hline \multirow[b]{2}{*}{ Scale/Score } & \multicolumn{2}{|c|}{$\mathbf{A D H D}^{\mathrm{a}}$} & \multicolumn{2}{|c|}{$\mathrm{NC}^{\mathbf{b}}$} & \multirow[b]{2}{*}{$d f$} & \multirow[b]{2}{*}{$t$} & \multirow[b]{2}{*}{$\eta^{2}$} \\
\hline & $M$ & $S D$ & $M$ & $S D$ & & & \\
\hline ADHD-IV Hyp.-Impulsive & 19.0 & 5.7 & 5.5 & 2.4 & 30.3 & $-10.3^{*}$ & .70 \\
\hline ADHD-IV Inattentive & 20.4 & 4.6 & 5.2 & 2.5 & 34.6 & $-13.9^{*}$ & .81 \\
\hline ADHD-IV Total & 39.4 & 9.1 & 10.7 & 4.4 & 32.4 & $-13.5^{*}$ & .80 \\
\hline Pretest SSRT & 434 & 105 & 311 & 90 & 47.0 & $-4.37 *$ & .28 \\
\hline
\end{tabular}

Note: $\mathrm{ADHD}=$ attention deficit hyperactivity disorder-combined type; $\mathrm{NC}=$ non-ADHD controls; $\eta^{2}=$ eta-squared effect size $\left(\mathrm{SS}_{\mathrm{effect}} /\right.$ $\left.\mathrm{SS}_{\text {total }}\right) ; \mathrm{ADHD}-\mathrm{IV}=\mathrm{ADHD}$ Rating Scale-IV; SSRT $=$ Stop signal reaction tim.

$\mathrm{a}_{n}=26 . \mathrm{b}_{n}=23$.

$* p<.001$. 
mean delay time. The measure of inhibition (SSRT) was computed by subtracting the mean delay time from the mean reaction time averaged across all four trials. The more difficulty participants had inhibiting their response, the higher their SSRT score would be. Thus, higher SSRT scores are associated with greater disinhibition.

Consistent with previous SST research and in support of participant groupings, boys in the ADHD group scored significantly higher pretest SSRT scores than comparison boys (see Table 1). In addition, the moderate effect size found for this sample is consistent with the combined effects size $(d=.64)$ reported in Oosterlaan et al.'s (1998) meta-analysis of SST studies that compared children with and without ADHD. These SST performance data also suggest ADHD participants' medication effects were sufficiently diminished.

\section{Competitive Puzzle Task}

An emotion-regulation task adapted from Hinshaw and Melnick (1995) formed the basis for the competitive puzzle task. Each boy was informed he would win a prize if he constructed his Lego $₫$ model faster than his competitor (an alleged boy in the next room). However, unbeknown to participants, their puzzle was insoluble due to missing pieces. Thus, the alleged competitor always won the race. A video camera and monitor were clearly visible to each participant at all times during the puzzle task. Boys were told,

The boy who you'll be racing against for the bonus prize is in the next room, and you can see and hear each other while working on your puzzles, but you are not allowed to talk to each other during the task. Remember, only the boy who solves the puzzle first will get the bonus prize.

The actual purpose of the camera was to record participants' behavior to be coded later for emotional reactivity and behavior regulation. The monitor displayed a videotaped child actor completing an identical puzzle with ease. The actor made self-directed statements such as "Boy, this is really easy!" and "I'm gonna win that prize!" to promote a competitive setting.

Emotion control condition. Before the puzzle race began, half of ADHD and half of control participants were told to self-regulate their behavior so as to mask any frustration emotion that may ensue during their puzzle assembly attempt (emotion control condition). Specifically, boys in the emotion control condition were told:

In fact, while you are solving your puzzle as quickly as you can, I want you to act like it is really easy to solve even if you are having difficulty. That way, the other boy won't know if you are having any trouble. So if the puzzle is hard for you, don't let the other boy know.

The other half of ADHD and control participants (no emotion control condition) were not given any additional instruction beyond what all boys were told.

Credibility of confederate video. Prior to data collection, 46 undergraduate students, unaware of the experimental design or intent of the study, rated the 3-min video clip of the child actor on a scale of 1 (strongly agree) to 5 (strongly disagree) across several dimensions. The majority of observers agreed or strongly agreed that the boy (i.e., child actor) was involved in competition $(97 \%)$, bragging $(85 \%)$, and solving his puzzle with ease (80\%). In addition, $87 \%$ of raters agreed the video represented a realistic and believable competitive situation.

Coding of videotaped emotion regulation. To measure each participant's effectiveness at emotion regulation, two trained undergraduate students, blind to the experimental hypotheses and conditions under study, coded videotapes from the Lego ${ }^{\circledR}$ task portion of the session. Videotapes of the 3-min puzzle session were event-coded, based on 5-sec intervals, for the presence of facial, behavioral, or vocal displays of emotion regulation according to a coding scheme adapted from Maedgen and Carlson (2000). Scores were derived from a frequency count of behaviors occurring in mutually exclusive subcategories: Emotional reaction was coded as either (a) No Emotional Expression (i.e., no facial, behavioral, or vocal displays of emotion), (b) Mild Emotional Expression (e.g., downturned mouth as in frown or grimace, makes a gesture of disappointment, grunts, or verbally acknowledges his frustration), or (c) Moderate Emotional Expression (e.g., slams fist, whines loudly, cries). Emotion regulation was coded as either (a) Task-Oriented (the boy attempts to regulate himself by making verbal self-reassuring statements or behaving in a way that indicates acceptance of the situation, such as trying to complete the puzzle despite missing pieces or search for missing pieces), (b) Negative Responses (the boy makes statements focusing on the negative or uncontrollable aspects of the task or displays disruptive behavior such as throwing puzzle pieces, attempting to leave the room, rude remarks to the competitor), or (c) Shuts Down (the boy is temporarily immobilized and backs off from task demands, such as crosses arms and refuses to continue). In addition to interval coding of emotional reaction and regulation, a 4-point global rating borrowed from Hinshaw and Melnick (1995) measured overall intensity of emotional display and ef- 
fectiveness at emotion regulation across the entire coding session.

Interrater reliability. Two observers coded a common $20 \%$ of all cases to establish interrater reliability estimates. For the global ratings, alpha coefficients were calculated and revealed adequate reliability (emotional intensity $\alpha=.92$; effectiveness at regulation $\alpha=.68$ ). These reliability estimates are comparable to those reported in studies using similar methodologies (Hinshaw \& Melnick, 1995; Maedgen \& Carlson, 2000). Intraclass correlation coefficients revealed satisfactory levels of consistency between observers for Task-Oriented $(r=.92)$, Negative Behavior $(r=.86)$, No Emotional Expression $(r=.84)$, and Mild Expression $(r=.74)$. Moderate Expression $(r=.44)$ and Shuts Down $(r=.39)$, however, were dropped from analyses due to inadequate reliability and low base rate occurrence.

\section{Procedure}

Each participant was scheduled for an individual, 1-hr laboratory session held on a university campus. Upon arrival at the lab, each boy's parent completed the ADHD-IV Rating Scale with one investigator while a second investigator briefly described the tasks and obtained verbal assent for participation from the participant. The boy was then directed to a computer station to complete the SST (practice trials plus four blocks of test trials) as a pretest measure of behavioral inhibition. After completing the SST, the boy was directed to an unassembled Lego® model on a table and directions for the puzzle race were read. Investigators waited outside the room during the competitive puzzle task. After 3 min elapsed and the videotaped actor had successfully completed his puzzle, an investigator returned and immediately directed the participant to the computer to repeat the SST. Investigators were instructed not to discuss the puzzle race with the participant and, if necessary, to say, "It's really important that we finish this computer game quickly, then we can talk about the Lego® race." Following posttesting, investigators debriefed each participant, explaining that some Lego $®$ pieces were missing. They apologized for the omission and explained the reason behind the deception and the true intent of the study. Once debriefing was complete, each boy was encouraged to successfully complete the puzzle and was given the bonus prize.

\section{Results}

\section{Data Reduction}

Because the four remaining event-coded observation variables fell into mutually exclusive categories (i.e., No Emotional Reaction vs. Mild Reaction and Task-Oriented vs. Negative Behavior) and because event-coded variables were expected to be related to global ratings, intercorrelations among all remaining observation variables were examined to check for redundancy. As anticipated, the variables were significantly intercorrelated, with correlations ranging from .78 to .99 . Thus, to create a composite measure of emotion dysregulation, Mild Emotional Expression, Negative Behavior, Global Emotional Intensity, and Global Effectiveness at Emotion Regulation (reverse coded) were combined into a total score to represent a total measure of observed emotion dysregulation. Cronbach's $\alpha$ revealed adequate reliability $(\alpha=.84)$, and this combination variable was used in all subsequent analyses.

\section{Predicting Emotion Regulation From Pretest SSRT}

As reported in Table 1, boys in the ADHD group earned significantly higher pretest SSRT scores than did comparison boys, $t(47)=-4.37, p<.001, \eta^{2}=.28$. To examine whether baseline behavioral disinhibition predicted emotion dysregulation during competition, a linear regression was performed with pretest SSRT, condition, and a Pretest SSRT $\times$ Condition interaction term entered by forward selection. The condition variable and interaction term were entered into the analysis because half the sample was given instruction to hide their emotional display and half were not; however, neither the condition nor the interaction term met the minimum criterion for model entry, and thus both were excluded as predictors in the model summary. Examination of normal probability plots of standardized residuals, as well as scatterplots of the Studentized residuals against predicted values, revealed no outliers and appropriate fit of the model. The resulting regression model, with pretest SSRT as a predictor of the combined emotion dysregulation score, was statistically significant, $F_{1,42}=6.29, p<.02$, with pretest SSRT accounting for $11 \%$ of the variance, $R^{2}$ adj $=.11$, in observed emotion dysregulation.

\section{Emotion Dysregulation by Group Status and Control Condition}

The effects of group and condition on emotion dysregulation during the competitive puzzle task were analyzed with a 2 (group: ADHD vs. comparison) $\times 2$ (condition: emotion control vs. no emotion control) analysis of variance. Results, presented in Table 2, revealed a significant main effect for group, with boys in the ADHD group rated more dysregulated than comparison boys. No statistically significant effects of condition or group-by-condition interaction resulted. However, it is possible this was due to small sample 
Table 2. Stop Signal Reaction Time Scores and Observed Emotion Dysregulatoin by Group and Condition

\begin{tabular}{|c|c|c|c|c|c|c|c|c|c|c|}
\hline \multirow[b]{2}{*}{ Variable } & \multicolumn{2}{|c|}{ ADHD } & \multicolumn{2}{|c|}{ NC } & \multicolumn{6}{|c|}{$\boldsymbol{F}$} \\
\hline & $M$ & $S D$ & $M$ & $S D$ & Group & $\eta_{p}^{2}$ & Condition & $\eta_{p}^{2}$ & $\begin{array}{l}\text { Group by } \\
\text { Condition }\end{array}$ & $\eta_{p}^{2}$ \\
\hline SSRT pretest & 434 & 105 & 311 & 90 & $t=-4.37 * *$ & .28 & NA & NA & NA & NA \\
\hline SSRT posttest & & & & & $8.81 * *$ & .18 & 0.03 & .00 & 1.26 & .03 \\
\hline Emotion control $^{\mathrm{a}}$ & 463 & 118 & 350 & 74 & & & & & & \\
\hline No emotion control ${ }^{\mathrm{b}}$ & 433 & 76 & 383 & 107 & & & & & & \\
\hline SSRT Proportion Change & & & & & $3.59 *$ & .08 & 2.40 & .06 & $4.96 * *$ & .11 \\
\hline Emotion control $^{\mathrm{a}}$ & 0.15 & 0.24 & 0.11 & 0.10 & & & & & & \\
\hline No emotion control ${ }^{\mathrm{b}}$ & 0.08 & 0.20 & 0.51 & 0.64 & & & & & & \\
\hline Emotion dysregulation & & & & & $6.56 * *$ & .14 & 1.96 & .05 & 0.63 & .02 \\
\hline Emotion control $^{\mathrm{a}}$ & 16.15 & 11.12 & 6.45 & 4.96 & & & & & & \\
\hline No emotion control ${ }^{\mathrm{b}}$ & 17.90 & 11.75 & 12.80 & 9.78 & & & & & & \\
\hline
\end{tabular}

Note: $\mathrm{ADHD}=$ attention deficit hyperactivity disorder-combined type; $\mathrm{NC}=$ non-ADHD controls; $\mathrm{SSRT}=$ stop signal reaction time; $\eta_{p}{ }^{2}=$ partial eta-squared effect size $\left(\mathrm{SS}_{\text {effect }} /\left[\mathrm{SS}_{\text {effect }}+\mathrm{SS}_{\text {error }}\right]\right)$.

${ }^{\mathrm{a}} n=13$ for ADHD; $n=12$ for NC. ${ }^{\mathrm{b}} n=13$ for ADHD; $n=11$ for NC.

$* p=.06 . * * p<.05$.

sizes rather than actual negligible mean differences. As such, effect sizes statistics, which reflect the proportion of variance explained by the independent variables, are also reported to clarify preliminary findings and to inform future research. Per Cohen's (1988) standards $\left(\eta^{2}\right.$ of .01 to .05 is small, .06 to .13 is moderate, and .14+ is large), effect size statistics revealed that group status represented a large effect size $\left(\eta_{p}^{2}=.15\right)$. emotion control condition $\left(\eta_{p}^{2}=.05\right)$ and the interaction between group and condition $\left(\eta_{p}^{2}=.02\right)$ accounted for small amounts of variance in emotion dysregulation. Figure 1 plots mean emotion dysregulation scores by group and condition and suggests that comparison boys who were not prompted to hide their emotional display had higher dysregulation scores during the frustrating task than did comparison boys who were instructed to control their emotional display.

\section{Effect of Emotion Dysregulation on Posttest SSRT}

To determine if group status or attempts to mask emotion during the frustrating competition task resulted in changes in SSRT performance from pre- to posttest, a 2 (group: ADHD vs. comparison) $\times 2$ (condition: emotion control vs. no emotion control) analysis of variance was applied to SSRT proportion change scores. Results of the analysis are presented in Table 2. Proportion change scores (each participant's simple SSRT posttest-pretest difference, divided by pretest SSRT) examined each boy's pre- to posttest change in SSRT while adjusting for baseline differences. The analysis of variance yielded a trend for group differences $(p=.06)$, with an effect size $\left(\eta_{p}^{2}=.08\right)$ suggesting that group status explained a moderate amount of variance in proportion change scores. Specifically, comparison boys showed more pre- to posttest proportional change in SSRT than boys in the ADHD group.
However, a significant group-by-condition interaction, representing a moderate effect size $\left(\eta_{p}{ }^{2}=.11\right)$, revealed it was the comparison boys in the no emotion control condition who exhibited the most change in SSRT performance (see Figure 2). In this figure, slopes depict the amount of proportional change from pre- to posttest, not actual levels of disinhibition. Examination of means revealed a significant increase in pre- to posttest SSRT scores for comparison boys in the no emotion control condition, whereas pre- and posttest SSRT scores for the emotion control group of comparison boys showed little change. For boys with ADHD, absolute SSRT scores remained significantly higher than non-ADHD boys at posttest, and pre- to posttest change was minimal and unaffected by emotion control condition.

\section{Discussion}

This study investigated boys' regulation of emotional reaction during frustrating peer competition and the relation between emotion regulation and levels of executive behavioral inhibition. Behavioral disinhibition, according to Barkley (1997), is the key to understanding the myriad of social difficulties associated with ADHD. He posited that because children with ADHD have deficits in behavioral inhibition, they also will have great difficulty restricting or keeping private their emotional reactions to evocative situations. Although existing research clearly supports the notion that behavioral disinhibition is a defining characteristic of ADHD, evidence is inconclusive as to its centrality in explaining secondary ADHD-related deficits, such as emotion dysregulation (Nigg, 2001). Among this sample of school-age boys with and without ADHD, we found that precompetition behavioral disinhibition scores predicted a small but statistically significant 


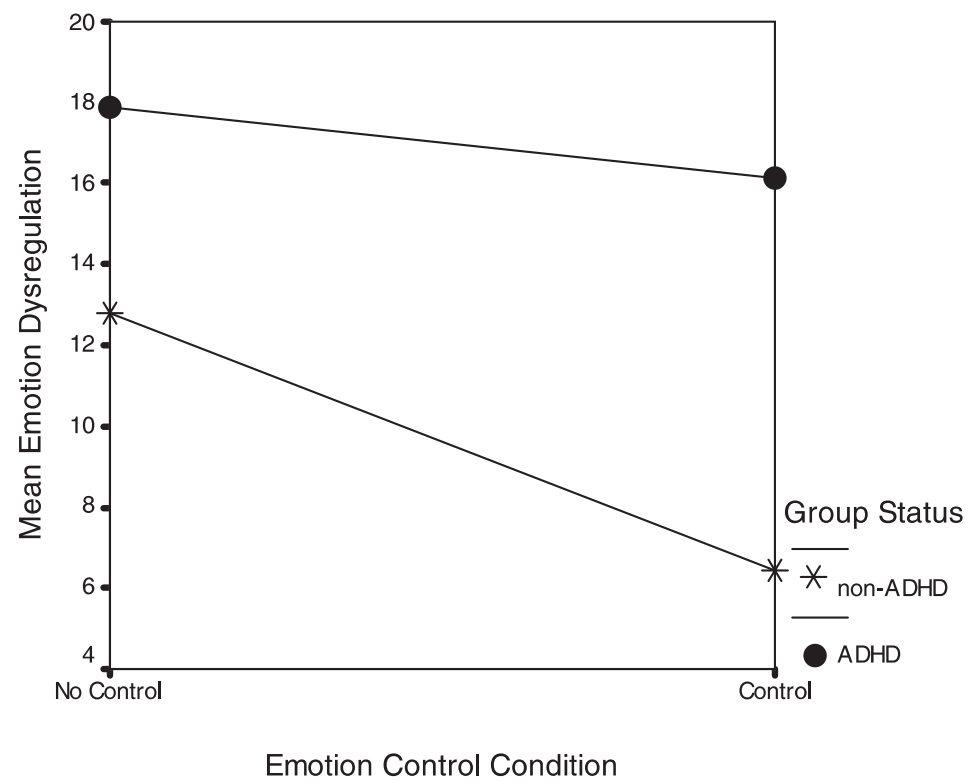

Figure 1. Mean emotion dysregulation by group and condition.

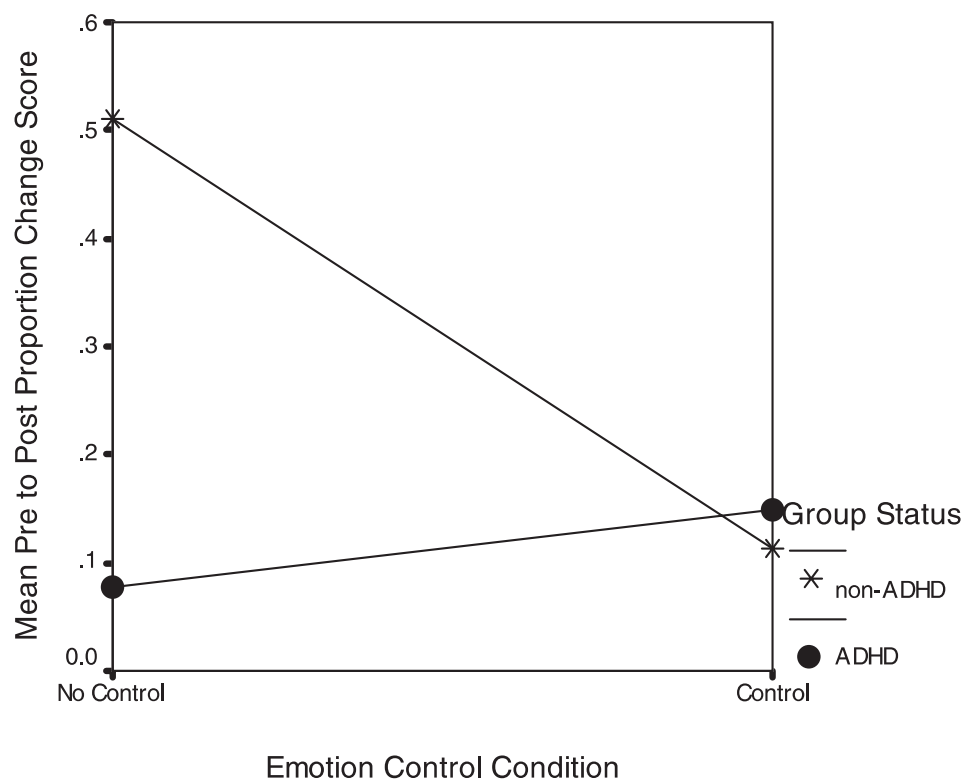

Figure 2. Mean SSRT proportional change by group and condition.

amount of variability in emotion dysregulation during frustrating peer competition. This suggests, however, that other significant mediators of emotion dysregulation exist as well. Comorbid aggression is one likely mediator that was not considered in this investigation (see Hinshaw \& Melnick, 1995). Future work should explore the contribution of aggression to our understanding of emotion dysregulation among children with ADHD.

Consistent with previous research, examination of group differences revealed that boys with ADHD were more ineffective at emotion regulation during the frustration task than were nonsymptomatic boys. Indeed, boys with ADHD demonstrated consistently high levels of emotion dysregulation even when directly instructed to control their emotional expression in front of their competitor. Non-ADHD comparison boys were better regulated overall, and a small group-by-condition effect size suggested that the subsample of comparison boys told to control their emotional displays in front of their competitor had even fewer instances of emotion dysregulation than those given no such in- 
struction. Thus, it appears comparison boys in this sample were effective at controlling their public displays of emotion with purposeful effort. The fact that unprompted comparison boys (with a mean age of 9 years, 2 months) did not spontaneously control their emotional displays to the same degree as prompted boys is not surprising, as emotion regulation is a developing skill for this age group (Zeman \& Garber, 1996). Although this effect size evidence is only preliminary, it does suggest the situational demand for restrained emotional expression may be an age-appropriate expectation for comparison boys, but not those with ADHD. If boys with ADHD were trying to regulate their emotional display when instructed, their efforts were not successful.

To confirm this possible performance deficit, future research should consider the nature of this apparent deficiency in emotion regulation among boys with ADHD. If boys with ADHD do attempt self-regulation when frustrated, are their emotion-control strategies qualitatively different than those used by non-ADHD peers? For example, data indicate the verbal mediation strategies (i.e., private speech) used by boys with ADHD for self-regulation purposes, especially in the face of increasing academic challenge, are immature and relatively ineffective (Berk \& Landau, 1993; Landau \& Berk, 2001). However, no known study has examined which (if any) particular strategies these children use when they are expected to control proscribed emotional outbursts. Future investigations should also contrast the notion that boys with ADHD possess fewer self-regulation strategies versus the possibility that they are unwilling or unmotivated to attempt emotion regulation when frustrated. For example, one might wonder if extreme reward dependence may have caused boys with ADHD in this study to focus exclusively on the prospect of gaining the bonus prize while ignoring the task of emotion regulation (see Carlson \& Tamm, 2000).

The final purpose of this study was to examine the short-term effects of observed emotion dysregulation during frustrating competition on subsequent inhibitory control (i.e., posttest performance on the SST). Because children with ADHD appear to have difficulty modulating their emotions and tend to become stuck in response patterns (Landau \& Milich, 1988), it was predicted that boys with ADHD would show little pre- to posttest change in level of behavioral disinhibition regardless of explicit prompts to mask emotional display during competition. In contrast, we expected that comparison boys told to self-regulate their emotional displays during competition would show improved inhibitory control at posttest (i.e., lower SSRT scores). As predicted, boys with ADHD had significantly higher pretest and posttest disinhibition scores than did comparison boys, and their disinhibition scores changed little from pretest to posttest. Thus, ADHD boys not only displayed consistent emotion dysregulation during the frustration task, they also showed an enduring pattern of behavioral disinhibition before and after the frustration task. The comparison group showed a different pattern of findings. Specifically, comparison boys who were not explicitly cued to self-regulate showed significantly more pre- to posttest SSRT change (see Figure 2), whereas SSRT scores for the prompted comparison group were relatively consistent before and after the frustration task. Thus, it was the comparison boys not prompted to control their emotions who showed greater disinhibition relative to baseline following the frustrating competition.

Although the unprompted comparison boys displayed more emotional dysregulation during the frustration task and were more disinhibited at posttest than their prompted peers, it is important to note that their levels of emotion dysregulation and behavioral disinhibition did not reach the levels found with the ADHD group. However, this pattern of findings among the comparison boys suggests a relation between emotion dysregulation and subsequent behavior disinhibition for this non-ADHD group. An important path for future research will be to examine how attempts to self-regulate frustration may have helped the subsample of prompted comparison boys to maintain their inhibitory control after frustration. That is, when one is frustrated, does successful emotion regulation serve to buffer what would otherwise interfere with inhibitory control?

Some limitations of this study are noteworthy. Due to small sample size and other restrictions of this sample (i.e., racially homogeneous/predominantly White and boys with ADHD who were receiving medication), our findings need to be replicated with larger samples. Second, there was no consideration of the effect of aggressive tendencies on either disinhibition or emotion dysregulation. Although there is evidence the inhibitory deficits of ADHD exist independent of comorbid aggression (Nigg, 1999), disentangling the role of aggression as it relates to emotion dysregulation is an essential direction for future research. This seems particularly necessary as only a small portion of variance in dysregulation was accounted for by disinhibition. Third, this study examined emotion regulation in a contrived context. As is always the case with laboratory studies, the credibility of the analog and effects of the lab setting on performance must be considered. Care was taken to ensure that the frustration paradigm seemed realistic, but examination of disinhibition and self-regulation of emotion within a naturalistic environment would assure that these findings apply to actual child experience. Finally, many conclusions about ADHD were derived from a failure to find significant performance differences as a function of the emotion control condition. Although this lack of response to condition was anticipated for boys with ADHD, infer- 
ences based on the null should always be interpreted with caution.

In summary, results of this investigation provide preliminary evidence for a small but significant relation between executive behavior disinhibition and subsequent deficits in self-regulation of emotion among school-age boys. We also found evidence that emotion dysregulation may negatively influence later executive control unless attempts to self-regulate emotion are employed. What best differentiated groups of boys in this study was the ability of boys without ADHD to respond successfully to prompts to self-regulate. Boys with ADHD showed no change in emotion regulation or disinhibition in response to self-regulation cues. The nature of this apparent performance deficit calls for further study as does the potentially reciprocal relation between emotion dysregulation and executive behavior inhibition.

\section{References}

American Psychiatric Association. (1987). Diagnostic and statistical manual of mental disorders (3rd ed., rev.). Washington, DC: Author.

American Psychiatric Association. (1994). Diagnostic and statistical manual of mental disorders (4thed.). Washington, DC: Author.

Barkley, R. A. (1997). Behavioral inhibition, sustained attention, and executive functions: Constructing a unifying theory of ADHD. Psychological Bulletin, 121, 65-94.

Berk, L. E., \& Landau, S. (1993). Private speech of learning disabled and normally achieving children in classroom academic and laboratory contexts. Child Development, 64, 556-571.

Biederman, J., Newcorn, J., \& Sprich, S. (1991). Comorbidity of attention deficit hyperactivity disorder with conduct, depressive, anxiety, and other disorders. American Journal of Psychiatry, 148, 564-577.

Carlson, C. L., \& Tamm, L. (2000). Responsiveness of children with attention-deficit/hyperactivity disorder to reward and response cost: Differential impact on performance and motivation. Journal of Consulting and Clinical Psychology, 68, 73-83.

Cohen, J. (1988). Statistical power analysis for the behavioral sciences (2nd ed.). Hillsdale, NJ: Lawrence Erlbaum Associates, Inc.

Cole, P. M., Michel, M. K., \& Teti, L. O. (1994). The development of emotion regulation and dysregulation: A clinical perspective. Monographs of the Society for Research in Child Development, 59, 73-100.

Cole, P. M., Zahn-Waxler, C., \& Smith, D. (1994). Expressive control during a disappointment: Variations related to preschoolers' behavior problems. Developmental Psychology, 30, 835-846.

DuPaul, G. J., Power, T. J., Anastopoulos, A. D., \& Reid, R. (1998). ADHD Rating Scale-IV-Checklists, norms, and clinical interpretations. New York: Guilford.

Eisenberg, N., Fabes, R. A., Gutherie, I. K., Murphy, B. C., Maszk, P., Holmgren, R., et al. (1996). The relations of regulation and emotionality to problem behavior in elementary school children. Development and Psychopathology, 8, 141-162.

Gaub, M., \& Carlson, C. L. (1997). Gender differences in ADHD: A meta-analysis and critical review. Journal of the American Academy of Child \& Adolescent Psychiatry, 36, 1036-1045.

Guevremont, D. C., \& Dumas, M. C. (1994). Peer relationship problems and disruptive behavior disorders. Journal of Emotional and Behavior Disorders, 2, 164-172.
Henker, B., \& Whalen, C. K. (1999). The child with attention-deficit/hyperactivity disorder in family contexts. In H. C. Quay \& A. E. Hogan (Eds.), Handbook of disruptive behavior disorders (pp. 139-155). New York: Plenum.

Hinshaw, S. P., \& Melnick, S. M. (1995). Peer relationships in boys with attention-deficit hyperactivity disorder with and without comorbid aggression. Development and Psychopathology, 7, 627-647.

Landau, S., \& Berk, L. E. (2001, XXXXX). Private speech and academic problem-solving in children with ADHD: Implications for classroom behavior. Poster presented to the National Association of School Psychologists Annual National Convention, Washington, DC.

Landau, S., \& Milich, R. (1988). Social communication patterns of attention-deficit-disordered boys. Journal of Abnormal Child Psychology, 16, 69-81.

Landau, S., Milich, R., \& Diener, M. B. (1998). Peer relations of children with attention-deficit hyperactivity disorder. Reading and Writing Quarterly, 14, 83-105.

Logan, G. D., Cowan, W. B., \& Davis, K. A. (1984). On the ability to inhibit simple and choice reaction time responses: A model and a method. Journal of Experimental Psychology: Human Perception and Performance, 10, 276-291.

Logan, G. D., Schachar, R. J., \& Tannock, R. (1997). Impulsivity and inhibitory control. Psychological Science, 8, 60-64.

Maedgen, J. W., \& Carlson, C. L. (2000). Social functioning and emotional regulation in the attention deficit hyperactivity disorder subtypes. Journal of Clinical Child Psychology, 29, 30-42.

Nigg, J. T. (1999). The ADHD response inhibition deficit as measured by the stop task: Replication with $D S M-I V$ combined type, extension, and qualification. Journal of Abnormal Child Psychology, 27, 393-402.

Nigg, J. T. (2000). On inhibition/disinhibition in developmental psychopathology: Views from cognitive and personality psychology and a working inhibition taxonomy. Psychological Bulletin, 126, 200-246.

Nigg, J. T. (2001). Is ADHD an inhibitory disorder? Psychological Bulletin, 127, 571-598.

Nigg, J. T., Blaskey, L., Huang-Pollock, C., \& Rappley, M. D. (2002). Neuropsychological executive functions and ADHD DSM-IV subtypes. Journal of the American Academy of Child \& Adolescent Psychiatry, 41, 59-66.

Oosterlaan, J., Logan, G. D., \& Sergeant, J. A. (1998). Response inhibition in $\mathrm{AD} / \mathrm{HD}, \mathrm{CD}$, Comorbid $\mathrm{AD} / \mathrm{HD}+\mathrm{CD}$, anxious, and control children: A meta-analysis of studies with the stop task. Journal of Child Psychology and Psychiatry, 39, 411-425.

Pelham, W. E. (1993). Pharmacotherapy for children with attention deficit hyperactivity disorder. School Psychology Review, 22,199-227.

Pennington, B. F., \& Ozonoff, S. (1996). Executive function and developmental psychopathology. Journal of Child Psychology and Psychiatry \& Allied Disciplines, 37, 51-87.

Pliszka, S. R., Borcherding, S. H., Spratley, K., Leon, S., \& Irick, S. (1997). Measuring inhibitory control in children. Developmental and Behavioral Pediatrics, 18, 254-259.

Power, T. J., Costigan, T. E., Leff, S. S., Eiraldi, R. B., \& Landau, S. (2001). Assessing ADHD across settings: Contributions of behavioral assessment to categorical decision making. Journal of Clinical Child Psychology, 30, 399-412.

Quay, H. C. (1997). Inhibition and attention deficit hyperactivity disorder. Journal of Abnormal Child Psychology, 25, 7-13.

Saarni, C. (1999). The development of emotional competence. New York: Guilford.

Sanson, A., Smart, D., Prior, M., \& Oberklaid, F. (1993). Precursors of hyperactivity and aggression. Journal of American Academy of Child \& Adolescent Psychiatry, 32,1207-1216.

Schachar, R., Mota, V. L., Logan G. D., Tannock, R., \& Klim, P. (2000). Confirmation of an inhibitory deficit in attention-defi- 
cit/hyperactivity disorder. Journal of Abnormal Child Psychology, 28, 227-235.

Shields, A., \& Cicchetti, D. (2001). Parental maltreatment and emotion dysregulation as risk factors for bullying and victimization in middle childhood. Journal of Clinical Child Psychology, 30, 349-363.

Shields, A., Cicchetti, D., \& Ryan, R. M. (1994). The development of emotional and behavioral self-regulation and social compe- tence among maltreated school-age children. Development and Psychopathology, 6, 57-75.

Zeman, J., \& Garber, J. (1996). Display rules for anger, sadness, and pain: It depends on who is watching. Child Development, 67, 957-973.

Received June 30, 2003

Accepted April 22, 2004 